\title{
Efficacy and Safety of Extracranial Vein Angioplasty in Multiple Sclerosis A Randomized Clinical Trial
}

Paolo Zamboni, MD; Luigi Tesio, MD; Stefania Galimberti, PhD; Luca Massacesi, MD; Fabrizio Salvi, MD; Roberto D'Alessandro, MD; Patrizia Cenni, MD; Roberto Galeotti, MD; Donato Papini, PhD; Roberto D’Amico, PhD; Silvana Simi, PhD; Maria Grazia Valsecchi, PhD; Graziella Filippini, MD; for the Brave Dreams Research Group

IMPORTANCE Chronic cerebrospinal venous insufficiency (CCSVI) is characterized by restricted venous outflow from the brain and spinal cord. Whether this condition is associated with multiple sclerosis (MS) and whether venous percutaneous transluminal angioplasty (PTA) is beneficial in persons with MS and CCSVI is controversial.

OBJECTIVE To determine the efficacy and safety of venous PTA in patients with MS and CCSVI.

DESIGN, SETTING, AND PARTICIPANTS We analyzed 177 patients with relapsing-remitting MS; 62 were ineligible, including 47 (26.6\%) who did not have CCSVI on color Doppler ultrasonography screening. A total of 115 patients were recruited in the study timeframe. All patients underwent a randomized, double-blind, sham-controlled, parallel-group trial in $6 \mathrm{MS}$ centers in Italy. The trial began in August 2012 and concluded in March 2016; data were analyzed from April 2016 to September 2016. The analysis was intention to treat.

INTERVENTIONS Patients were randomly allocated (2:1) to either venous PTA or catheter venography without venous angioplasty (sham).

MAIN OUTCOMES AND MEASURES Two primary end points were assessed at 12 months: (1) a composite functional measure (ie, walking control, balance, manual dexterity, postvoid residual urine volume, and visual acuity) and (2) a measure of new combined brain lesions on magnetic resonance imaging, including the proportion of lesion-free patients. Combined lesions included T1 gadolinium-enhancing lesions plus new or enlarged T2 lesions.

RESULTS Of the included 115 patients with relapsing-remitting MS, 76 were allocated to the PTA group (45 female [59\%]; mean [SD] age, 40.0 [10.3] years) and 39 to the sham group (29 female [74\%]; mean [SD] age, 37.5 [10.6] years); 112 (97.4\%) completed follow-up. No serious adverse events occurred. Flow restoration was achieved in 38 of 71 patients (54\%) in the PTA group. The functional composite measure did not differ between the PTA and sham groups ( $41.7 \%$ vs $48.7 \%$; odds ratio, $0.75 ; 95 \% \mathrm{Cl}, 0.34-1.68 ; P=.49$ ). The mean (SD) number of combined lesions on magnetic resonance imaging at 6 to 12 months were 0.47 (1.19) in the PTA group vs 1.27 (2.65) in the sham group (mean ratio, 0.37; 95\% Cl, 0.15-0.91; $P=.03$ : adjusted $P=.09$ ) and were $1.40(4.21)$ in the PTA group vs 1.95 (3.73) in the sham group at 0 to 12 months (mean ratio, $0.72 ; 95 \% \mathrm{Cl}, 0.32-1.63 ; P=.45$; adjusted $P=.45$ ). At follow-up after 6 to 12 months, 58 of 70 patients (83\%) in the PTA group and 22 of 33 (67\%) in the sham group were free of new lesions on magnetic resonance imaging (odds ratio, 2.64; $95 \% \mathrm{Cl}, 1.11-6.28 ; P=.03$; adjusted $P=.09)$. At 0 to 12 months, 46 of 73 patients (63.0\%) in the PTA group and 18 of $37(49 \%)$ in the sham group were free of new lesions on magnetic resonance imaging (odds ratio, $1.80 ; 95 \% \mathrm{Cl}, 0.81-4.01 ; P=.15$; adjusted $P=.30$ ).

CONCLUSION AND RELEVANCE Venous PTA has proven to be a safe but largely ineffective technique; the treatment cannot be recommended in patients with MS.

TRIAL REGISTRATION clinicaltrials.gov Identifier: NCTO1371760

JAMA Neurol. 2018;75(1):35-43. doi:10.1001/jamaneurol.2017.3825

Published online November 18, 2017.
Editorial page 15

Supplemental content
Author Affiliations: Author affiliations are listed at the end of this article.

Group Information: The members of the Brave Dreams Research Group are listed at the end of the article.

Corresponding Author: Paolo Zamboni, MD, Translational Surgery and Vascular Diseases Centre, University of Ferrara Hospital, Via Aldo Moro 8, 44124 Cona, Ferrara, Italy (paolozamboni@icloud.com). 
W hen intraluminal defects, compression, or hypoplasia are identified in the internal jugular or azygos veins, the condition is known as chronic cerebrospinal venous insufficiency (CCSVI). ${ }^{1,2}$ An Italian open-label study ${ }^{3}$ published in 2009 including 65 patients with multiple sclerosis (MS) found an association between MS and CCSVI. The study also found that venous percutaneous transluminal angioplasty (PTA) was associated with an improved Multiple Sclerosis Functional Composite (MSFC) score at 1 year in patients with relapsing-remitting MS (RRMS) and a reduction in the proportion of those with gadolinium-enhancing lesions on brain magnetic resonance imaging (MRI). This preliminary study attracted considerable media attention in Italy and elsewhere; media-based groups formed to promote the treatment, and some called to make it freely available. ${ }^{4}$

Subsequent to the initial reports by Zamboni et $\mathrm{al},{ }^{3}$ prevalence studies of CCSVI in MS have reported conflicting results. A small 2012 case-control study ${ }^{5}$ further assessed the potential of venous PTA to benefit patients with MS. One year after venous PTA, the study found that functional score was improved compared with baseline, and the authors suggested that a double-blind randomized trial was justified. ${ }^{5}$

Both the National Institute for Health and Care Excellence and the Italian Ministry of Health have urged that randomized clinical trials be conducted to assess the efficacy of venous PTA for CCSVI in MS. ${ }^{6,7}$ In response to these calls and to public pressure, the Directorate-General for Health and Welfare of the Italian Region of Emilia Romagna funded the Brain Venous Drainage Exploited Against Multiple Sclerosis (Brave Dreams) trial to investigate the efficacy and safety of venous PTA in patients with MS and CCSVI. We report the results of that trial.

\section{Methods}

\section{Study Design}

The Brave Dreams trial was a multicenter, randomized, doubleblind, sham-controlled, parallel-group trial to evaluate the efficacy and safety of venous PTA in patients with MS and CCSVI in extracranial or extravertebral veins. The study was conducted at 6 MS centers in Italy and their associated color Doppler ultrasonography (ECD) and angiography units, all of which were accredited by the Italian National Health Service. The Brave Dreams steering committee appointed an End Points Commission, which issued a detailed operations manual and trained trial physicians (who assessed primary functional outcomes, operated the ECD equipment, and performed catheter venography without venous angioplasty and venous PTA). After training, the commission issued an accreditation that was necessary for physicians to participate in the trial. Study monitoring was delegated to the company Medical Trials Analysis Italy in Ferrara, Italy. A trial data coordinating center was established to oversee data collection and quality and to perform the statistical analyses. The study adhered to the Helsinki Declaration and the International Council for Harmonisation of Technical Requirements for Pharmaceuticals for Human Use Good Clinical Practice guidelines and was approved by the ethical committee of the University of

\section{Key Points}

Question What is the efficacy of venous percutaneous transluminal angioplasty (PTA) for chronic cerebrospinal venous insufficiency in patients with multiple sclerosis?

Findings In the Brave Dreams trial, which included 115 patients with relapsing-remitting multiple sclerosis, venous PTA did not increase the proportion of patients who improved functionally nor did it reduce the mean number of new combined brain lesions on magnetic resonance imaging at 12 months. However, there was a tendency for more patients to become free of new lesions after venous PTA mainly because of a reduction in new lesions appearing 6 to 12 months after randomization.

Meaning Venous PTA cannot be recommended for patients with relapsing-remitting multiple sclerosis.

Ferrara Hospital and subsequently by the ethical committees of the other participating centers. The trial protocol has been published. ${ }^{8}$ The trial protocol can be found in Supplement 1. All participants provided written informed consent.

\section{Participants}

Patients were recruited at the participating centers. Eligibility criteria included age 18 to 65 years; a diagnosis of RRMS, according to the $2005 \mathrm{McDonald}$ criteria ${ }^{9}$; a diagnosis of secondary progressive MS, according to Lublin and Reingold ${ }^{10}$; care provided by the recruiting center for at least 2 years; at least 1 relapse in RRMS in the 2 years prior to enrollment; a baseline Expanded Disability Status Scale (EDSS) score of 2 to $5.5^{11}$; a disease duration of 15 years or less at baseline; stable neurological condition without relapse for at least 30 days before baseline; CCSVI, as determined by ECD examination carried out in accordance with a screening protocol ${ }^{12}$; and not receiving MS-specific treatment, immunomodulating, or immunosuppressive therapy without changes for at least 6 months up to baseline. Patients were ineligible if they had previous venous PTA or had received fingolimod therapy, cladribine therapy, laquinimod therapy, botulinum toxin therapy, infusion pump or neurostimulator implantation, or had participated in any clinical trial within 3 months of baseline. The complete list of exclusion criteria was published previously. ${ }^{8}$

\section{Randomization and Blinding}

The data coordinating center set up an internet-based computerized central randomization protocol stratified by participating center with variable length blocks, which assigned patients to the PTA or sham group in a 2:1 ratio. Treatment assignment was made known to the treating surgeon (via the electronic case report form) only on the day of the operation. Patients, all other study investigators, and operating room and hospital personnel were blinded to assignment.

To maintain patient blinding, surgeons were trained to deliver a catheter venography intervention that simulated venous PTA. ${ }^{8}$ This involved sudden acceleration of the catheter as it passed through the internal jugular vein together with a comment from the radiologist suggesting that venous PTA had been performed. 


\section{Procedures}

Participants underwent catheter venography without venous angioplasty of the azygos and internal jugular veins, with percutaneous access via the left femoral vein. The presence and location of CCSVI was assessed as reported elsewhere. ${ }^{2,3,5}$ If venography was positive for CCSVI, participants randomized to the PTA group received venous PTA during the venography session. If CCSVI was absent, those assigned to the PTA group received catheter venography without venous angioplasty. Those allocated to the sham group received catheter venography without venous angioplasty. These procedures were performed via day surgery. Overnight hospital stay was never required in this trial. All patients received prophylactic low-molecular-weight heparin during the 3 subsequent weeks.

\section{Outcomes}

There were 2 primary end points at 12 months: a functional end point and an MRI end point.

\section{Functional End Point}

In view of the psychometric limitations of the EDSS and the MSFC scores, ${ }^{13,14}$ the steering committee decided to use a new primary composite end point based on a range of the functional impairments commonly experienced by patients with MS. This composite included walking control, balance, manual dexterity, postvoid residual urine volume, and visual acuity (eMethods in Supplement 2). Statistically significant changes were adopted for defining changes in walking control, balance, and manual dexterity; a minimal real difference was used for postvoid residual urine volume and visual acuity. ${ }^{15}$ Based on the changes found, each index was considered improved, stable, or worsened. Patients were classified as (1) improved, which indicated improvement in 1 or more functions and stability in the remaining functions, (2) worsened, which indicated worsening of 1 or more functions and stability in nonworsening functions, (3) mixed, which indicated presence of improved and worsened functions, or (4) stable, which indicated no change in any function. Evaluations were performed by operators (2 per participating center) at baseline, within 15 days of venography, and 3, 6, and 12 months later. Only results at 12 months were used in analyses. To ensure the reliability of the functional measurements, the End Points Commission made 1 or 2 visits to each site to monitor the functional assessments. Assessors were required to make video recordings of the walking control and manual dexterity tests in $10 \%$ or more of patients and to send them to the commission for inspection.

If a patient had a transient impairment that blocked performance of 1 or more functional tests, these were not performed until the next follow-up. In the event of a clinical relapse, all tests were deferred. As per study protocol, the final round of tests could take place up to 15 months after venography.

\section{Magnetic Resonance Imaging End Point}

Scans were acquired at baseline and at 6 and 12 months after venography. The primary MRI end point was the number of new combined cerebral lesions at 12 months compared with baseline. New combined lesions included (1) new lesions on T2-weighted images, (2) preexisting lesions enlarged by greater than $30 \%$ on T2-weighted images, and (3) gadolinium- enhancing lesions in T1-weighted images of preexisting lesions. An additional MRI end point was the proportion of patients free of new lesions. Before starting the study, each center acquired a set of MRI scans of a single individual on 2 separate occasions. The scans were assessed by the Department of Neurosciences at the University of Florence for image quality, repositioning accuracy, and signal-to-noise ratio to check that quality was sufficient. At each center, MRIs were always performed with the same device (at least $1.5 \mathrm{~T}$ ) and with the same protocol. The scans were assessed by an experienced specialist blinded to treatment assignment.

\section{Secondary Outcomes}

Secondary outcomes at 12 months were the proportion of patients with CCSVI diagnosed by ECD but not confirmed by venography, annualized relapse rate, change in EDSS score, proportion of patients with relapses, and proportion of patients who had venous PTA with restored flow on ECD at 12 months.

\section{Safety}

The types and grade of adverse events are reported according to the Good Clinical Practice guidelines. Stopping rules included serious adverse events and/or a false-positive rate for CCSVI on ECD exceeding $10 \% .^{8}$

\section{Statistical Analysis}

To achieve a $90 \%$ power ( $\mathrm{SD}=7.6$ ) to detect a hypothesized 2.1 fewer active MRI lesions in the PTA group (3.9 vs 6.0), 423 patients with RRMS were needed. This number of patients was also sufficient (92\% power) to detect a hypothesized $15 \%$ more patients in the PTA group than the sham group improving on the functional end point ( $a=.05 ; 2$-sided test on proportions). Patients in the sham group were themselves expected to improve by $15 \%$ over the year of follow-up. Because both primary end points were used to characterize treatment benefit, each null hypothesis had to be rejected at the same significance level ( $a=.05$ ), with multiplicity adjustment only for the MRI end point, since this had 2 parameters (mean lesion number and proportion of lesion-free patients). ${ }^{16}$

Because of low enrollment ( 6 patients per month by November 2013), the protocol was amended in February 2014; 300 patients with RRMS (200 in PTA group and 100 in sham group) were required to reveal the same hypothesized differences in MRI and functional end points, with powers of $80 \%$ and $84 \%$, respectively. The study closed in December 2014 because of slow recruitment, with 115 patients with RRMS recruited (38.3\% of amended target).

The initial power calculation for patients with secondary progressive MS indicated that 222 patients were needed. ${ }^{8}$ Recruitment was stopped in February 2014, with only 15 patients recruited. Stopping decisions were taken without knowledge of outcomes or treatment allocations.

For descriptive purposes, we calculated percentage, mean (SD), and median (interquartile range), as appropriate. The main analysis was intention to treat. The effect of PTA vs sham on the composite functional end point was assessed by comparing the proportions of improved patients at 1 year in the 2 groups, and the significance of differences in proportion was assessed by $x^{2}$ test. The effect of PTA vs sham on the MRI end point was assessed 
Figure. Study Flowchart for Patients With Relapsing-Remitting Multiple Sclerosis

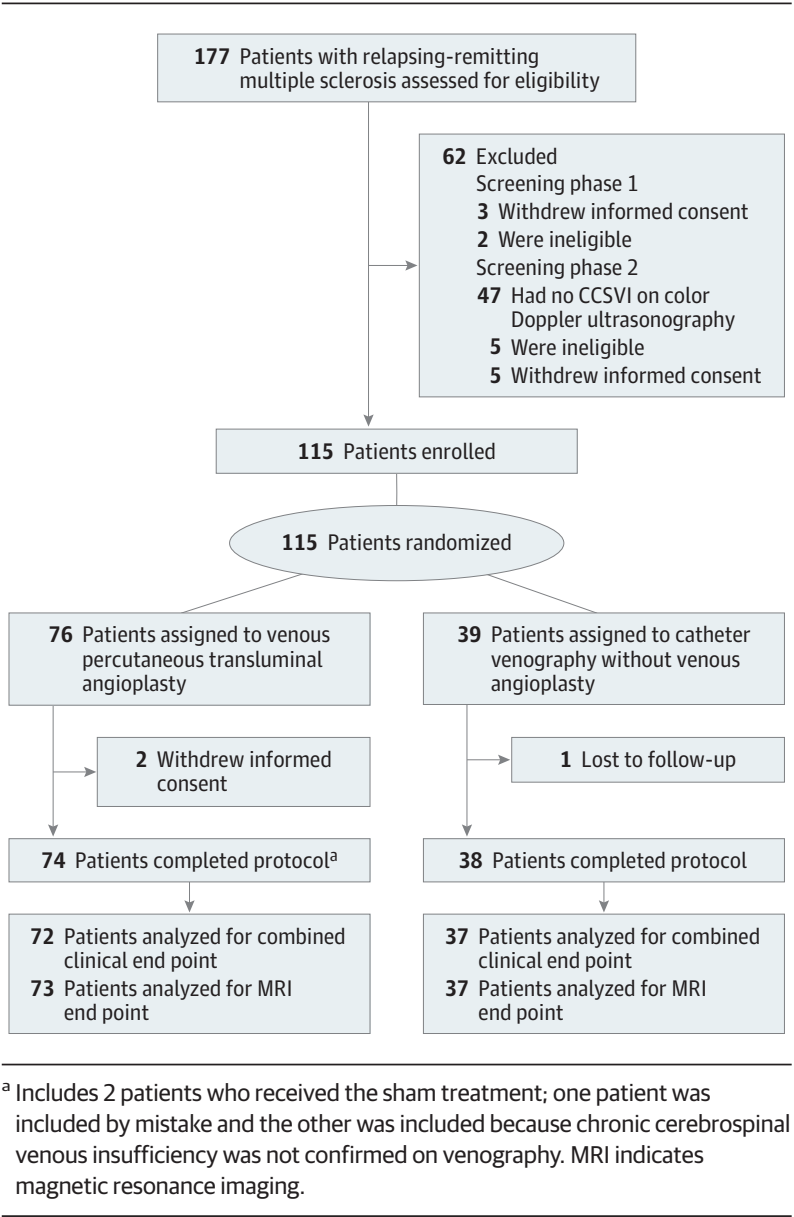

by a negative binomial model that compared the mean number of MRI lesions in the 2 groups at 1 year. The proportion of patients who were free of new brain lesions on MRI were compared by $x^{2}$ test. Variables that were unbalanced at baseline were adjusted for using a logistic model on the functional end point and a negative binomial model on mean number of MRI lesions.

Relapse rates (secondary end point) in the 2 groups were compared assuming a Poisson distribution of events. Differences in EDSS scores at 1 year were compared by analysis of covariance testing, with adjustment for baseline scores. When analyzing components of the functional and MRI end points (including findings at 0 to 6 and 6 to 12 months), we adjusted for multiplicity using the Hommel method, ${ }^{17}$ since it is reasonable to assume that variables involved could be directly related (reported as adjusted $P$ value). All tests were 2 -tailed, with the significance level set at $P<.05$. Analyses were performed with SAS version 9.3 (SAS Institute) and R version 3.2 (R Foundation for Statistical Computing).

\section{Results}

The Figure shows the study flowchart for patients with RRMS. A total of 177 were assessed for eligibility, and 62 were ineli-
Table 1. Baseline Characteristics of Recruited Patients and Their Disease According to Treatment Group

\begin{tabular}{|c|c|c|}
\hline \multirow[b]{2}{*}{ Characteristic } & \multicolumn{2}{|l|}{ №. (\%) } \\
\hline & $\begin{array}{l}\text { PTA } \\
(n=76)\end{array}$ & $\begin{array}{l}\text { Sham } \\
(\mathrm{n}=39)\end{array}$ \\
\hline Female & $45(59)$ & $29(74)$ \\
\hline Age, mean (SD), y & $40.0(10.3)$ & $37.5(10.6)$ \\
\hline \multicolumn{3}{|l|}{ EDSS score } \\
\hline 2 or 2.5 & $50(66)$ & $24(62)$ \\
\hline 3 or 3.5 & $18(24)$ & $11(28)$ \\
\hline 4 or 4.5 & $8(11)$ & $2(5)$ \\
\hline 5 or 5.5 & 0 & $2(5)$ \\
\hline Median (interquartile range) & $2.5(2.0-3.0)$ & $2.5(2.0-3.5)$ \\
\hline Mean (SD) & $2.6(0.7)$ & $2.7(0.9)$ \\
\hline $\begin{array}{l}\text { Years since MS diagnosis, median } \\
\text { (interquartile range) }\end{array}$ & $4.3(2.8-8.4)$ & $6.1(3.7-9.0)$ \\
\hline \multicolumn{3}{|l|}{ Relapses in previous 2 years, No. } \\
\hline 0 & 0 & 0 \\
\hline 1 & $44(58)$ & $26(67)$ \\
\hline 2 & $24(32)$ & $4(10)$ \\
\hline$\geq 3$ & $8(11)$ & $9(23)$ \\
\hline Median (interquartile range) & $1.0(1.0-2.0)$ & $1.0(1.0-2.0)$ \\
\hline Mean (SD) & $1.7(1.2)$ & $1.8(1.4)$ \\
\hline \multicolumn{3}{|l|}{$\begin{array}{l}\text { Intraluminal obstacle at ECD } \\
\text { in at least } 1 \mathrm{IJV}\end{array}$} \\
\hline Yes & $74(97)$ & $37(95)$ \\
\hline No & $2(3)$ & $2(5)$ \\
\hline \multicolumn{3}{|l|}{$\begin{array}{l}\text { Bidirectional and/or absent flow } \\
\text { at ECD in at least } 1 \mathrm{IJV} \text {, in } 2 \text { positions }\end{array}$} \\
\hline Yes & $70(92)$ & $38(97)$ \\
\hline No & $6(8)$ & $1(3)$ \\
\hline \multicolumn{3}{|l|}{ T1 gadolinium-enhancing lesions, No. } \\
\hline Median (range) & $0(0-8)$ & $0(0-3)$ \\
\hline Mean (SD) & $0.49(1.2)$ & $0.23(0.58)$ \\
\hline \multicolumn{3}{|l|}{ Immunomodulatory therapy } \\
\hline Yes & $31(41)$ & $18(46)$ \\
\hline No & $45(59)$ & $21(54)$ \\
\hline
\end{tabular}

Abbreviations: ECD, color Doppler ultrasonography; EDSS, Expanded Disability Status Scale; IJV, internal jugular vein; MS, multiple sclerosis; PTA, percutaneous transluminal angioplasty.

gible, including 47 (26.6\%) who did not have CCSVI on ECD screening. One hundred fifteen patients were eligible and randomly assigned to the PTA group ( $n=76$ ) or the sham group ( $n=39$ ), which included catheter venography without venous angioplasty, between August 7, 2012, and December 15, 2014. The 2 groups were similar for baseline characteristics except that patients in the sham group had more women and longer disease duration (Table 1). No serious adverse events attributable to catheter venography or venous PTA occurred, but 2 adverse events (1.7\%) did occur: 1 vagal reaction and 1 episode of transient neck pain. A total of 112 of 115 patients (97.4\%) completed the 12-month follow-up, with similar proportions completing in the 2 groups. eFigure 1 in Supplement 2 shows the study flowchart for patients with secondary progressive MS.

\section{Primary End Points}

Functional end point results were available for 109 patients with RRMS (Table 2). A total of 30 of 73 patients (41\%) in the PTA group and 18 of 37 (49\%) in the sham group improved on 


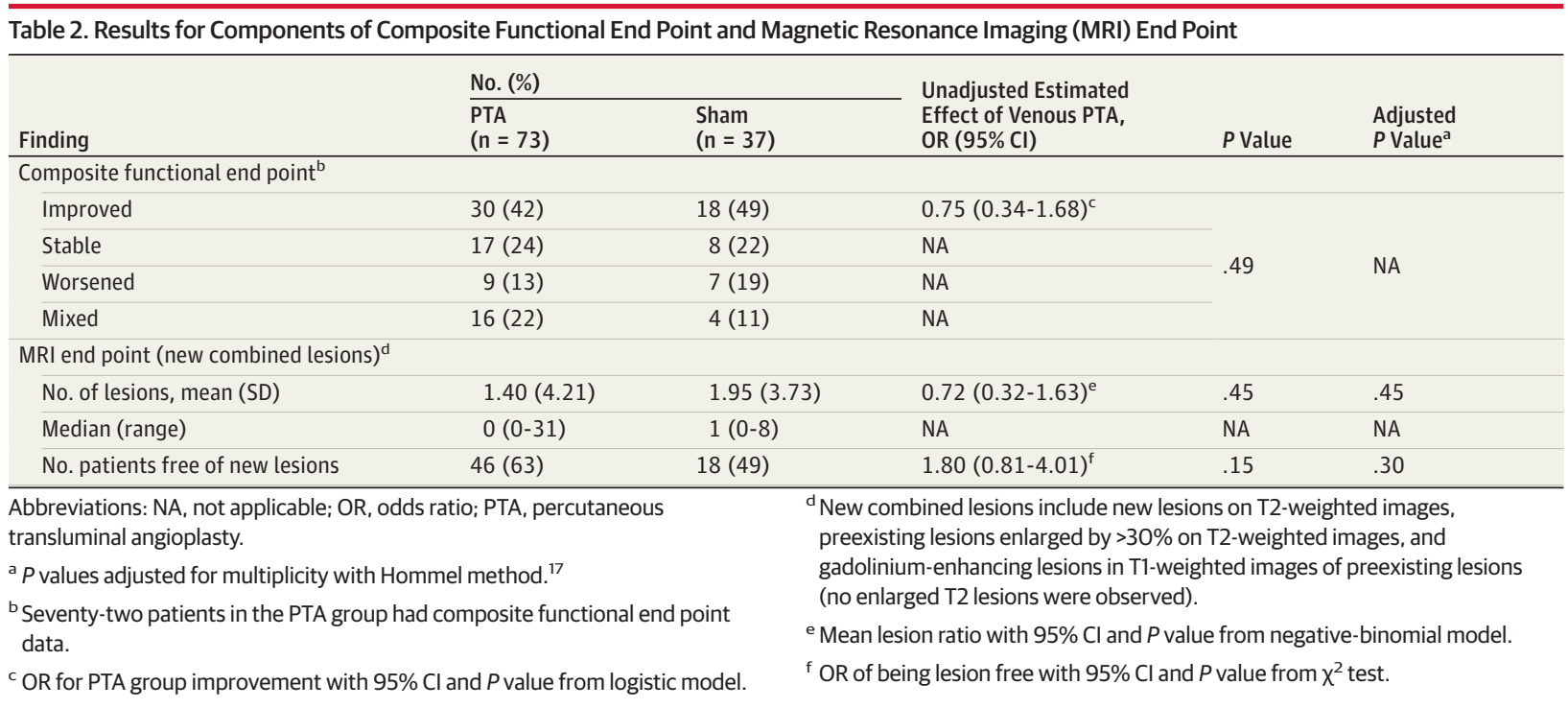

the functional end point-a difference of $-7 \%$ (95\% CI, -26.7 to 10.1) in favor of the sham group. The logistic model adjusted for sex and disease duration provided an odds ratio (OR) for improvement in the PTA group (compared with the sham group) of 0.70 (95\% CI, 0.31-1.59; $P=.40$ ). The OR for the unadjusted model was 0.75 (95\% CI, 0.34-1.68; $P=.49)$. Worsening occurred in 9 patients (12\%) in the PTA group vs 7 (19\%) in the sham group; functional stability was maintained in 17 (23\%) in the PTA group and 8 (22\%) in the sham group. A mixed outcome (improvement in one or more functions and worsening in one or more) occurred in 16 patients (22\%) in the PTA group and 4 (11\%) in the sham group. Results for individual components of the functional end point are shown in Table 3. More patients in the PTA group than in the sham group improved in visual acuity and manual dexterity, while more patients in the PTA group worsened for postvoid residual urine volume and balance. Walking control remained stable in most patients of both groups.

The number of new lesions on MRI at 12 months compared with baseline was unrelated to venous PTA; there were a mean 1.40 new lesions in the PTA group vs 1.95 in the sham group (mean ratio, 0.72; 95\% CI, 0.32-1.63; $P=.45$; adjusted $P=.45$ ) (Table 2). In the regression analysis adjusted for sex and disease duration, the mean PTA-to-sham ratio was 0.50 (95\% CI, 0.20-1.27; $P=.30$ ). The proportion of patients free of new lesions did not differ significantly between the PTA and sham groups (63.0\% vs 48.6\%; OR, 1.80; 95\% CI, 0.81-4.00; $P=.15$; adjusted $P=.30$ ) (Table 2). At 12 months, 50 patients $(68 \%)$ in the PTA group were free of new or enlarged T2 lesions compared with 21 (57\%) in the sham group (OR, 1.66; 95\% CI, 0.73-3.75; $P=.22$; adjusted $P=.62$ ), and $53(73 \%)$ in the PTA group were free of gadolinium-enhancing lesions at 12 months compared with 18 (49\%) in the sham group (OR, 2.76; 95\% CI, 1.14-6.68; $P=.02$; adjusted $P=.08$ ) (Table 4).

A post hoc analysis investigating new brain lesions at 0 to 6 months (eTable 1 in Supplement 2) and at 6 to 12 months found that at 6 to 12 months, 58 of 70 patients (83\%) in the PTA group and 22 of 33 (67\%) in the sham group (OR, 2.64; 95\% CI, 1.11-
6.28; $P=.03$; adjusted $P=.09$ ) were free of combined brain lesions, while there were a mean (SD) 0.36 (0.98) new T2 lesions in the PTA group and 1.14 (2.63) in the sham group $(P=.02$; adjusted $P=.06)$. Finally, 61 patients $(87 \%)$ in the PTA group and 24 (73\%) in the sham group (OR, 2.75; 95\% CI, 1.10-6.89; $P$ = .03; adjusted $P=.09$ ) were free of new T2 lesions (Table 4 ).

\section{Secondary End Points}

Eight of 115 patients with RRMS (7.0\%) diagnosed as having CCSVI by ECD had no abnormalities on venography, so the positive predictive value of ECD was $93.0 \%$ in patients with RRMS. The annualized relapse rate was 0.32 (95\% CI, 0.2-0.4) in the PTA group and 0.39 (95\% CI, 0.2-0.5) in the sham group, giving a relative rate of $0.82(95 \% \mathrm{CI}, 0.40-1.71 ; P=.60)$. The median (interquartile range) baseline EDSS score was 2.5 (2.03.0) in the PTA group and 2.5 (2.0-3.5) in the sham group (Table 1). At 12 months, the median (interquartile range) EDSS score was 2.0 (1.5-3.0) in the PTA group and 2.0 (1.5-2.5) in the sham group $(P=.49)$. Seventeen of 73 patients $(23 \%)$ in the PTA group had least 1 relapse over the 12 months compared with 12 of 39 (31\%) in the sham group. Blinded flow assessment at 12 months revealed restored flow in 38 of 71 patients (54\%) in the PTA group and 14 of 37 (38\%) in the sham group.

\section{Secondary Progressive MS}

Primary end points for the 15 patients with secondary progressive MS (10 in the PTA group and 5 in the sham group) are shown, in descriptive form only, in eTable 2 in Supplement 2.

\section{Discussion}

Venous PTA did not increase the proportion of patients with RRMS who improved on the functional composite measure compared with the sham procedure over the 12-month follow-up, nor did it significantly reduce the appearance of new combined brain lesions on MRI at 0 to 12 months. Expanded Disability Status Scale disability measures were 


\begin{tabular}{|c|c|c|c|c|c|c|c|c|}
\hline \multirow{2}{*}{$\begin{array}{l}\text { Functional } \\
\text { Assessment }\end{array}$} & \multirow{2}{*}{$\begin{array}{l}\text { Total } \\
\text { No. }\end{array}$} & \multicolumn{3}{|c|}{ At $12 \mathrm{mo}$, No. (\%) } & \multicolumn{2}{|l|}{ Baseline Score } & \multicolumn{2}{|l|}{$\underline{\text { Score at } 12 \mathrm{mo}}$} \\
\hline & & Improved & Stable & Worsened & Median (Range) & Mean (SD) & Median (Range) & Mean (SD) \\
\hline \multicolumn{9}{|c|}{ Visual Acuity (No. of Lines Read) } \\
\hline \multicolumn{9}{|c|}{$100 \%$ Contrast } \\
\hline PTA & 72 & $5(7)$ & $64(89)$ & $3(8)$ & $12.0(8.0-14.0)$ & $11.9(1.0)$ & $12.0(9.0-14.0)$ & $11.9(1.0)$ \\
\hline Sham & 37 & $1(3)$ & $33(89)$ & $3(4)$ & $12.0(6.0-14.0)$ & $11.9(1.6)$ & $12.0(6.0-14.0)$ & $11.6(1.7)$ \\
\hline \multicolumn{9}{|c|}{$2.5 \%$ Contrast } \\
\hline PTA & 72 & $6(8)$ & $61(85)$ & $5(7)$ & $8.0(3.0-10.0)$ & $7.5(1.3)$ & $8.0(5.0-10.0)$ & $7.64(1.2)$ \\
\hline Sham & 37 & $1(3)$ & $33(89)$ & $3(8)$ & $8.0(3.0-10.0)$ & $7.3(1.7)$ & $8.0(1.0-10.0)$ & $7.22(1.9)$ \\
\hline \multicolumn{9}{|c|}{$1.25 \%$ Contrast } \\
\hline PTA & 72 & $11(15)$ & $59(82)$ & $2(3)$ & $6(1.0-8.0)$ & $5.7(1.4)$ & $6.0(3.0-9.0)$ & $6.00(1.5)$ \\
\hline Sham & 37 & $4(11)$ & $30(81)$ & $3(8)$ & $6(0.0-8.0)$ & $5.3(2.0)$ & $5.0(0.0-10.0)$ & $5.41(2.3)$ \\
\hline \multicolumn{9}{|l|}{ Any contrast } \\
\hline PTA & 72 & $15(21)$ & $49(68)$ & $8(11)$ & NA & NA & NA & NA \\
\hline Sham & 37 & $5(14)$ & $27(73)$ & $5(14)$ & NA & NA & NA & NA \\
\hline \multicolumn{9}{|c|}{ Manual Dexterity (No. of Cubes Displaced) } \\
\hline \multicolumn{9}{|c|}{ Dominant hand } \\
\hline PTA & 72 & $13(18)$ & $55(76)$ & $4(6)$ & $67.3(38.0-81.0)$ & $64.5(10.1)$ & $68.3(40.5-89.5)$ & $67.7(10.1)$ \\
\hline Sham & 37 & $5(14)$ & $31(84)$ & $1(3)$ & $66.0(36.0-85.5)$ & $64.4(11.4)$ & $67.0(42.0-92.5)$ & $67.0(13.1)$ \\
\hline \multicolumn{9}{|c|}{ Nondominant hand } \\
\hline PTA & 72 & 14 (19) & $55(76)$ & $3(4)$ & $63.5(35.0-77.0)$ & $61.6(9.3)$ & $64.8(31.5-83.0)$ & $64.4(11.3)$ \\
\hline Sham & 37 & $6(16)$ & $30(81)$ & $1(3)$ & $61.0(42.5-86.0)$ & $62.4(10.9)$ & $63.0(38.0-89.0)$ & $64.8(12.8)$ \\
\hline \multicolumn{9}{|l|}{ Any hand } \\
\hline PTA & 72 & $18(25)$ & $50(69)$ & $4(6)$ & NA & NA & NA & NA \\
\hline Sham & 37 & $7(19)$ & $29(78)$ & $1(3)$ & NA & NA & NA & NA \\
\hline \multicolumn{9}{|c|}{ Postvoid Residual Urine Volume $(\mathrm{mL})$} \\
\hline PTA & 69 & $20(30)$ & $36(54)$ & $11(16)$ & $43.0(0.0-256.0)$ & $64.7(61.4)$ & $33.0(0.0-327.0)$ & $50.3(58.9)$ \\
\hline Sham & 36 & $11(31)$ & $22(61)$ & $3(8)$ & $54.0(0.0-263.0)$ & $73.2(69.1)$ & $35.5(0.0-277.0)$ & $57.3(64.4)$ \\
\hline \multicolumn{9}{|c|}{ Balance Test (\% Adherence to Path) } \\
\hline PTA & 72 & $16(22)$ & $51(71)$ & $5(7)$ & $84.0(46.0-109.0)$ & $82.5(12.0)$ & $88.5(54.0-102.0)$ & $85.0(10.2)$ \\
\hline Sham & 37 & $8(22)$ & $28(76)$ & $1(3)$ & $81.0(60.0-99.0)$ & $78.6(11.7)$ & $84.0(60.0-102.0)$ & $82.2(11.8)$ \\
\hline \multicolumn{9}{|c|}{ Walking Control (Walk Ratio ${ }^{\mathrm{b}}$ ) } \\
\hline PTA & 72 & 0 & $70(97)$ & $2(3)$ & $5.5(4.2-7.6)$ & $5.6(0.8)$ & $5.5(4.2-7.5)$ & $5.6(0.8)$ \\
\hline Sham & 37 & 0 & $36(97)$ & $1(3)$ & $5.4(3.7-7.3)$ & $5.3(0.8)$ & $5.3(3.6-7.1)$ & $5.3(0.8)$ \\
\hline \multicolumn{9}{|c|}{ Abbreviations: NA, not applicable; PTA, percutaneous transluminal angioplasty. } \\
\hline \multicolumn{9}{|c|}{ a All $P$ values were $>.99$ after adjustment for multiplicity. } \\
\hline patiof & lim & cton from & (non & & & & & \\
\hline
\end{tabular}

stable and similar between the groups at 12 months, matching the composite functional outcome. The annualized relapse rate was also similar between the groups. Safety data indicated no serious adverse events attributable to venous PTA or the sham procedure.

The functional outcome explored by the trial was reduction in disability as assessed by 5 functions (ie, walking control, balance, manual dexterity, postvoid residual urine volume, and visual acuity; eMethods in Supplement 2), which are the most frequent causes of disability in MS. The hypothesis that venous PTA can significantly reduce disability is rejected by findings from this study, which not only found no difference between the groups on the functional composite measure but also no difference for any of its 5 components (Table 3).

The primary MRI outcome measure was a difference in the number of new combined brain lesions. Venous PTA had no effect on this measure (Table 2). However, at 12 months, more than $20 \%$ of patients in the PTA group were free of gadoliniumenhancing lesions compared with the sham group (OR, 2.76;
95\% CI, 1.14-6.68) (Table 4). To explore this effect, which appears inconsistent with the other MRI data at 0 to 12 months, we performed an exploratory post hoc comparison of MRI findings at 0 to 6 months with those at 6 to 12 months (Table 4; eTable 1 in Supplement 2). We found a reduction in the mean number of new brain lesions (corresponding to more lesionfree patients) in the PTA group compared with the sham group at 6 to 12 months. The delayed and positive effect on the magnetic resonance biomarker suggests that PTA could affect the dynamic of the blood-brain barrier.

Gadolinium enhancement is a marker of damage to the blood-brain barrier, whose time course depends on lymphatic drainage ${ }^{18}$ and hence on venous drainage from the skull. ${ }^{19}$ Previous studies have reported that venous pressure is lowered ${ }^{3}$ and cerebrospinal fluid dynamics is improved ${ }^{20}$ after venous PTA, thereby favoring the drainage of cerebrospinal fluid into the dural veins, which depends on a pressure gradient between the subarachnoid spaces and dural veins. ${ }^{21,22}$ Another study ${ }^{23}$ reported that white matter lesion load was 


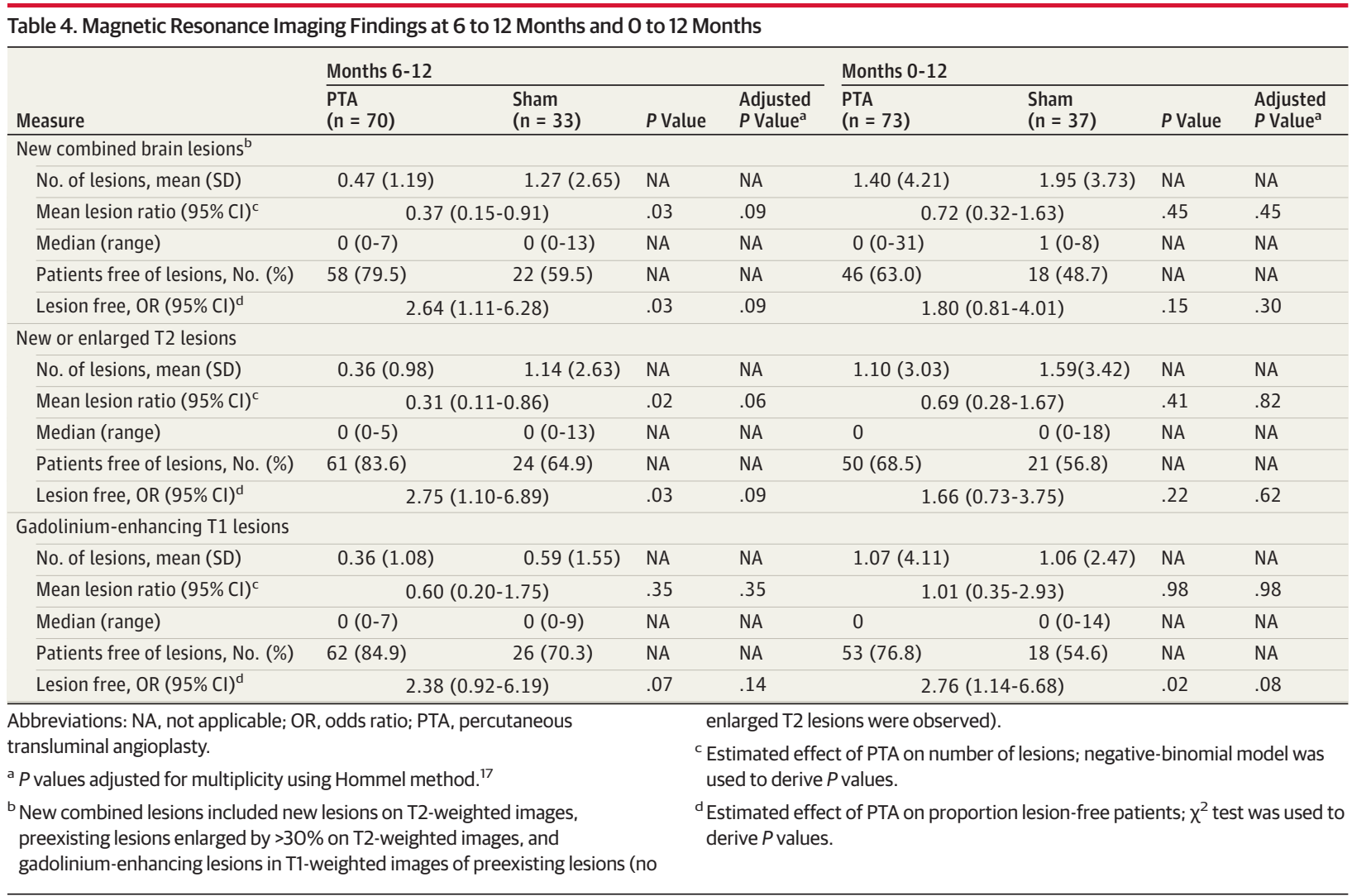

inversely correlated with reduced cerebrospinal fluid dynamics, as measured by MRI. In addition, flow improvement through the internal jugular veins owing to venous PTA has been reported to improve brain perfusion in patients with RRMS. ${ }^{21}$ It has also been reported that the development of a new MS plaque was preceded by sustained MRI-detected hypoperfusion before the plaque was identified on MRI. ${ }^{24,25}$

To our knowledge, few published data are available to compare with Brave Dreams findings. The positive effect of venous PTA on disability found in the first open-label pilot study $^{3}$ was not confirmed in the present study. The first study $^{3}$ was not blinded and used the MSFC and the EDSS scores as primary end points. There is some degree of subjectivity in assessing MSFC and EDSS scores, and with nonblinded patients and outcome assessors, detection bias may have been introduced.

In a sham-controlled randomized trial, ${ }^{26} 19$ patients with MS (9 assigned to PTA and 10 to sham) were assessed 1, 3, and 6 months after intervention. It was found that clinical and MRI end points were closely similar in the 2 groups. However, the study was flawed by the small number of recruited patients and short follow-up. ${ }^{26}$ In an observational study, ${ }^{27} 29$ patients with RRMS were observed for 2 years after venous PTA. A small improvement in mean EDSS score occurred over that time, and the mean annual relapse rate also lowered. Magnetic resonance imaging data were not reported.

\section{Limitations}

Our study has several limitations. Despite being, to our knowledge, the largest randomized study on venous PTA in RRMS, the power of the study was limited. There are at least 3 main reasons for this. First, many MS centers are reluctant to propose patients for a trial exploring a controversial hypothesis. Thus, of the 15 centers that initially joined the trial, only 6 actively recruited. Second, many patients are reluctant to participate in a randomized sham-controlled study in spite of the fact that randomization was 2:1 in favor of angioplasty; this reluctance may have been exacerbated by the media, which overemphasized the effectiveness of venous PTA. Third, the sudden widespread availability of venous PTA (and stenting) in private centers decreased the number of patients fulfilling the inclusion criterion of no previous angioplasty. A post hoc power calculation, based on control findings, indicated a power of $30 \%$ to detect a difference in the functional end point and $17 \%$ power to detect the target difference in the MRI end point. In terms of effect size and clinical relevance, the $95 \%$ CI for the estimated $7 \%$ advantage of sham varied from $26.7 \%$ in favor of sham to $10.1 \%$ in favor of PTA, thereby excluding the $15 \%$ target threshold for a benefit of venous PTA on the functional end point. However, the findings on new MRI lesions do not completely exclude an effect of venous PTA, since the 95\% CI for the mean lesion ratio of 0.72 in favor of PTA includes the 0.65 target for PTA improvement (95\% CI, 0.32-1.63).

The use of a new composite functional outcome measure was a major challenge. Although the outcomes and change thresholds (reflecting sensitivity to change) of the individual components have been validated (eMethods in Supplement 2), the composite is presented for the first time in this article.

Another limitation of the trial is that the severity of the enrolled patients in terms of EDSS score and lesion load was lower 
than anticipated, and patients with active disease may have been underrepresented in the study (Table 1). Finally, the fact that venous PTA was largely ineffective in restoring blood flow in nearly half the patients in the PTA group suggests that it was inadequate for exploring our initial hypothesis.

\section{Conclusions}

A number of neurologists and scientists expressed the opinion that the decision to conduct a trial on CCSVI in the absence of valid scientific evidence was unethical and a waste of resources. ${ }^{28}$ However, we believe that the best way to provide useful information to patients (and regulatory authorities) on the benefit and safety of venous PTA was to conduct a randomized trial-as also recommended by NICE $^{7}$ - that assessed outcomes directly relevant to patients. ${ }^{29}$ Venous PTA has proven to be a safe but ineffective technique in treating CCSVI in about half of patients. The procedure cannot be recommended for treatment of patients with MS; no further double-blinded clinical studies are needed. The delayed effect of venous PTA 6 months after the procedure on the magnetic resonance biomarker suggests a possibility that PTA may produce benefit for a subgroup of patients with MS. This should be further analyzed and investigated.

\section{ARTICLE INFORMATION}

Accepted for Publication: August 23, 2017.

Published Online: November 18, 2017 doi:10.1001/jamaneurol.2017.3825

Open Access: This is an open access article distributed under the terms of the CC-BY License. (C) 2017 Zamboni P et al. JAMA Neurology.

Author Affiliations: Translational Surgery and Vascular Diseases Centre, University of Ferrara Hospital, Ferrara, Italy (Zamboni); Department of Biomedical Sciences for Health, Chair of Physical and Rehabilitation Medicine, University of Milan, Milan, Italy (Tesio); Italian Auxologico Institute, Milan, Italy (Tesio); Center of Biostatistics for Clinical Epidemiology, School of Medicine and Surgery, University of Milan-Bicocca, Milan, Italy (Galimberti, Valsecchi); Department of Neurosciences Drugs and Child Health, University of Florence, Florence, Italy (Massacesi); Institute of the Neurological Science, Bellaria Hospital, Bologna, Italy (Salvi, D'Alessandro); Neuroradiology, Ravenna Hospital, Ravenna, Italy (Cenni); Interventional Radiology, University of Ferrara, Ferrara, Italy (Galeotti); Regional Agency for Health and Social Care, Regione Emilia-Romagna, Italy (Papini); Department of Diagnostic, Clinical and Public Health Medicine, University of Modena and Reggio Emilia, Modena, Italy (D'Amico); MS Cochrane Group. Institute of Clinical Physiology, Pisa, Italy (Simi); Scientific Director's Office, Carlo Besta Foundation and Neurological Institute, Milan, Italy (Filippini).

Author Contributions: Drs Galimberti and Valsecchi had full access to all of the data in the study and take responsibility for the integrity of the data and the accuracy of the data analysis. Study concept and design: Zamboni, Tesio, Galimberti, Salvi, D'Alessandro, Cenni, Papini, D’Amico, Simi, Valsecchi, Filippini.

Acquisition, analysis, or interpretation of data: Zamboni, Tesio, Galimberti, Massacesi, Cenni, Galeotti, D'Amico, Valsecchi, Filippini. Drafting of the manuscript: Zamboni, Tesio, Galimberti, Massacesi, Cenni, D'Amico, Valsecchi. Critical revision of the manuscript for important intellectual content: Zamboni, Tesio, Galimberti, Massacesi, Salvi, D'Alessandro, Galeotti, Papini, D'Amico, Simi, Valsecchi, Filippini.

Statistical analysis: Tesio, Galimberti, Massacesi, D'Amico, Valsecchi.

Obtained funding: Zamboni, D'Alessandro. Administrative, technical, or material support: Cenni, Galeotti, Papini.
Study supervision: Zamboni, Tesio, Massacesi D’Amico, Simi, Filippini.

Conflict of Interest Disclosures: Dr Zamboni was the main author of the articles first describing chronic cerebrospinal venous insufficiency and its association with multiple sclerosis as well as the hypothesis of the potential anti-inflammatory action of venous outflow restoration. Dr Massacesi has received honoraria for speaking in scientific meeting or for participating on advisory boards for Genzyme, Biogen, and Roche and has received travel support for scientific meetings from MerckSerono, Biogen, Teva, Genzyme, and Novartis. Dr Salvi is supported by a research grant from TEVA Company. No other disclosures were reported.

Funding/Support: This article was supported by the Regione Emilia-Romanga and the DirectorateGeneral for Health and Welfare as well as by contributing charities.

Role of the Funder/Sponsor: The funders had no role in the design and conduct of the study; collection, management, analysis, and interpretation of the data; and preparation, review, or approval of the manuscript. The trial steering committee had final responsibility for the decision to submit the manuscript for publication.

Group Information: The Brave Dreams Research Group members included the following: University of Ferrara Hospital, Ferrara, Italy: Stefano Ceruti, MD; Paolo Conforti, MD; Anna Maria Malagoni, MD, PhD; Erica Menegatti, PhD; Mirko Tessari, PhD; Francesca Pancaldi, BA; and Maria Elena Vanini, RT; Institute of Neurological Science, Bellaria Hospital, Bologna, Italy: Fabrizio Salvi, MD (PI); Elena Barbarossa, BA; Ilaria Bartolomei, MD; and Lisa Pellegrino, BA; S. Maria delle Croci Hospital, Ravenna, Italy: Maria Grazia Piscaglia, MD; Fabrizio Rasi, MD; Maria Babini, MD; Antonella Drea, MD; Eugenia Guerrini, MD; Enrico Maria Lotti, MD; Agnese Morelli, MD; Milena Peroni, MD; Valentina Zalambani, MD; and Sauro Zecchini, MD; Vittorio Emanuele Polyclinic University Hospital, Catania, Italy: Francesco Patti, MD; Clara Chisari, MD; Ignazio Chiaramonte, MD; Vincenzo Cimino, MD; Alessia Giaquinta, MD; Luigi Di Pino, MD; Gianni Failla, MD; and Pierfrancesco Veroux, MD; Maggiore della Carità Hospital, Novara, Italy: Roberto Cantello, MD; Maurizio Leone, MD; Lorenzo Coppo, MD; Giuseppe Guzzardi, MD; Olga Raymkulova, MD; Simona Ruggerone, MD; Alessandro Stecco, MD; and Domizia Vecchio, MD; C. Besta Neurological Institute and Foundation, Milan, Italy: Paolo Agostino Confalonieri, MD; Angela Campanella, MD; Valentina Caldiera, MD; Elisa Ciceri, MD; Alessandra
Erbetta, MD; Giuseppe Faragò, MD; Leila Parma, MD; Barbara Reggiori, MD; and Valentina Torri Clerici, MD; Ospedale Riuniti University Hospital, Ancona, Italy: Maura Danni, MD; Salvatore Arborino, MD; Fabiana De Berardinis, MD; Laura Di Biagio, MD; Chiara Orni, MD; Rosita Renzi, MD; Pamela Rosettani, MD; and Sara Zagaglia, MD; and Civitanova Marche Hospital, Civitanova Marche, Italy: Giuseppe Luccioni, MD; Luigi Oncini, MD; and Cristina Quatrini, MD. Additionally, the Brave Dreams trial was supported by the following groups: End Points Commission: Paolo Boldrini, MD (Department of Rehabilitative Medicine, Local Health Authority, Treviso, Italy); Roberto D'Amico, $\mathrm{PhD}$ (Statistics Institute, University of Modena and Reggio Emilia, Modena, Italy); Fabrizio Salvi, MD (Institute of the Neurological Science, Bellaria Hospital, Bologna, Italy); Silvana Simi, PhD (International MS Cochrane Group, Milan, Italy); and Luigi Tesio, MD (Department of Biomedical Sciences for Health, Chair of Physical and Rehabilitation Medicine, University of Milan, Milan, Italy); Accreditation Commission for Participating Centres: Patrizia Cenni, MD (Neuroradiology، Ravenna Hospital, Ravenna, Italy); Graziella Filippini, MD (Scientific Director's Office, Carlo Besta Neurological Institute and Foundation, Milan, Italy); Roberto Galeotti, MD (Interventional Radiology, University of Ferrara, Ferrara, Italy); and Paolo Zamboni, MD (Translational Surgery and Vascular Diseases Centre, University Hospital of Ferrara, Ferrara, Italy); Training and Coordinating Centre for Functional Measures: Luigi Tesio, MD (Department of Biomedical Sciences for Health, Chair of Physical and Rehabilitation Medicine, University of Milan, Milan, Italy); Training and Coordination Centre for Color Doppler Ultrasonography: Paolo Zamboni, MD (Translational Surgery and Vascular Diseases Centre, University Hospital of Ferrara, Ferrara, Italy); Contract Research Organization: Medical Trials Analysis Italy, Ferrara, Italy; Magnetic Resonance Imaging Working Group: Patrizia Cenni, MD (Neuroradiology. Ravenna Hospital, Ravenna, Italy); and Luca Massacesi, MD (Department of Neuroscience, University of Florence, Florence, Italy); Training Centre for Venography and Angioplasty: Patrizia Cenni, MD (Neuroradiology, Ravenna Hospital, Ravenna, Italy); and Roberto Galeotti, MD (Interventional Radiology, University of Ferrara, Ferrara, Italy); Centralized Magnetic Resonance Imaging Reading Centre: Luca Massacesi, MD (Department of Neuroscience, University of Florence, Florence, Italy); Data Coordinating Centre: Stefania Galimberti, PhD (Centre for Biostatistics in 
Clinical Epidemiology, University of Milano-Bicocca, Milan, Italy); and Maria Grazia Valsecchi, PhD (Centre for Biostatistics in Clinical Epidemiology, University of Milano-Bicocca, Milan, Italy); and Electronic Case Report Form Workgroup and Randomization: Stefania Galimberti, PhD (Centre for Biostatistics in Clinical Epidemiology, University of Milano-Bicocca, Milan, Italy); Graziella Filippini, MD (Scientific Director's Office, Carlo Besta Neurological Institute and Foundation, Milan, Italy); Medical Trials Analysis Italy, Ferrara, Italy; Luigi Tesio, MD (Department of Biomedical Sciences for Health, Chair of Physical and Rehabilitation Medicine, University of Milan, Milan, Italy); and Maria Grazia Valsecchi, PhD (Centre for Biostatistics in Clinical Epidemiology, University of MilanoBicocca, Milan, Italy).

Additional Contributions: We thank Don Ward, BSc (professional translator), for help with writing the manuscript in English and the late Alessandro Liberati, MD (Regional Agency for Health and Social Care, Regione Emilia-Romagna, Italy), for his major role in the development and actualization of the trial. Mr Ward was compensated for his contributions. We also thank the following charities for contributing to the trial: Associazione CCSVI nella Sclerosi Multipla, Associazione Sportiva Dilettanti, Associazione CCSVI Campania, Associazioni Sportive Italiane Ferrara, Centro Amministrativo Farmacie, Circolo Ricreativo Municipio Rovereto, Circolo Le Café Lenzi Davide, Circolo Officina Ferrarese del Motorismo Storico, Gruppo Calcistico Portomaggiore, Lions Club Ravenna Host, Lions Club Ferrara Host, Musei Arte Antica, Panathlon International Club di Taranto, Parrocchia Santi Pietro e Paolo, Proloco di Poggiorenatico, Rotaract Club Ferrara, SPAL Ferrara football team, Società Automatic Data Processing Inc Italia, Società Byte Software House, Unione Agricoltori Ferrara in Memoria lole Giglioli Gulinelli, and Università Terza Età Ferrara.

\section{REFERENCES}

1. Lee BB, Baumgartner I, Berlien P, et al; International Union of Phlebology. Diagnosis and treatment of venous malformations: consensus document of the International Union of Phlebology (IUP): updated 2013. Int Angiol. 2015;34(2):97-149.

2. Zivadinov R, Bastianello S, Dake MD, et al; International Society for Neurovascular Disease. Recommendations for multimodal noninvasive and invasive screening for detection of extracranial venous abnormalities indicative of chronic cerebrospinal venous insufficiency: a position statement of the International Society for Neurovascular Disease. J Vasc Interv Radiol. 2014; 25(11):1785-1794.e17.

3. Zamboni P, Galeotti R, Menegatti E, et al. A prospective open-label study of endovascular treatment of chronic cerebrospinal venous insufficiency. J Vasc Surg. 2009;50(6): 1348-1358.e1, 3.
4. Chafe R, Born KB, Slutsky AS, Laupacis A. The rise of people power. Nature. 2011;472(7344): 410-411.

5. Zamboni P, Galeotti R, Weinstock-Guttman B, Kennedy C, Salvi F, Zivadinov R. Venous angioplasty in patients with multiple sclerosis: results of a pilot study. Eur J Vasc Endovasc Surg. 2012;43(1):116-122.

6. Ministero della Salute Consiglio Superiore di Sanità. Trasmissione parere "Insufficienza venosa cerebro-spinale cronica (CCSVI) e sclerosi multipla (SM)." Roma, Italy: Sessione XLVIII; 2011.

7. National Institute for Health and Care Excellence. Percutaneous venoplasty for chronic cerebrospinal venous insufficiency for multiple sclerosis. https://www.nice.org.uk/guidance /ipg420. Accessed March 16, 2017.

8. Zamboni P, Bertolotto A, Boldrini P, et al; Chair of the Steering Committee. Efficacy and safety of venous angioplasty of the extracranial veins for multiple sclerosis: Brave Dreams study (Brain Venous Drainage Exploited Against Multiple Sclerosis): study protocol for a randomized controlled trial. Trials. 2012;13:183.

9. Polman $\mathrm{CH}$, Reingold $\mathrm{SC}$, Edan $\mathrm{G}$, et al. Diagnostic criteria for multiple sclerosis: 2005 revisions to the "McDonald criteria." Ann Neurol. 2005:58(6):840-846.

10. Lublin FD, Reingold SC; National Multiple Sclerosis Society (USA) Advisory Committee on Clinical Trials of New Agents in Multiple Sclerosis. Defining the clinical course of multiple sclerosis: results of an international survey. Neurology. 1996 ; 46(4):907-911.

11. Kurtzke JF. Rating neurologic impairment in multiple sclerosis: an Expanded Disability Status Scale (EDSS). Neurology. 1983;33(11):1444-1452.

12. Nicolaides AN, Morovic S, Menegatti E, Viselner G, Zamboni P. Screening for chronic cerebrospinal venous insufficiency (CCSVI) using ultrasound: recommendations for a protocol. Funct Neurol. 2011;26(4):229-248

13. Ebers GC, Heigenhauser L, Daumer M, Lederer C, Noseworthy JH. Disability as an outcome in MS clinical trials. Neurology. 2008;71(9):624-631.

14. Cohen JA, Reingold SC, Polman $\mathrm{CH}$, Wolinsky JS; International Advisory Committee on Clinical Trials in Multiple Sclerosis. Disability outcome measures in multiple sclerosis clinical trials: current status and future prospects. Lancet Neurol. 2012;11 (5):467-476.

15. Tesio L. Outcome measurement in behavioural sciences: a view on how to shift attention from means to individuals and why. Int J Rehabil Res. 2012;35(1):1-12.

16. European Agency for the Evaluation of Medicinal Products. Points to consider on multiplicity issues in clinical trials. http://www.ema.europa.eu/docs/en_GB/document
_library/Scientific_guideline/2009/09 /WC500003640.pdf. Accessed December 21, 2016.

17. Hommel G. A stagewise rejective multiple test procedure based on a modified Bonferroni test. Biometrika. 1988;75:383-386.

18. Gaitán MI, Shea CD, Evangelou IE, et al. Evolution of the blood-brain barrier in newly forming multiple sclerosis lesions. Ann Neurol. 2011; 70(1):22-29.

19. Iliff JJ, Lee $H$, Yu M, et al. Brain-wide pathway for waste clearance captured by contrast-enhanced MRI. J Clin Invest. 2013;123(3):1299-1309.

20. Zivadinov R, Magnano C, Galeotti R, et al. Changes of cine cerebrospinal fluid dynamics in patients with multiple sclerosis treated with percutaneous transluminal angioplasty: a case-control study. J Vasc Interv Radiol. 2013;24 (6):829-838

21. Zamboni $P$, Menegatti $E$, Cittanti $C$, et al. Fixing the jugular flow reduces ventricle volume and improves brain perfusion. J Vasc Surg Venous Lymphat Disord. 2016;4(4):434-445.

22. Bateman GA, Lechner-Scott J, Lea RA. A comparison between the pathophysiology of multiple sclerosis and normal pressure hydrocephalus: is pulse wave encephalopathy a component of MS? Fluids Barriers CNS 2016;13(1):18.

23. Magnano C, Schirda C, Weinstock-Guttman B, et al. Cine cerebrospinal fluid imaging in multiple sclerosis. J Magn Reson Imaging. 2012;36(4): 825-834.

24. Wuerfel J, Bellmann-Strobl J, Brunecker $P$, et al. Changes in cerebral perfusion precede plaque formation in multiple sclerosis: a longitudinal perfusion MRI study. Brain. 2004;127(pt 1):111-119.

25. Holland CM, Charil A, Csapo I, et al. The relationship between normal cerebral perfusion patterns and white matter lesion distribution in 1249 patients with multiple sclerosis. J Neuroimaging. 2012;22(2):129-136.

26. Siddiqui $A H$, Zivadinov R, Benedict RH, et al. Prospective randomized trial of venous angioplasty in MS (PREMiSe). Neurology. 2014;83(5):441-449.

27. Salvi F, Bartolomei I, Buccellato E, Galeotti R, Zamboni P. Venous angioplasty in multiple sclerosis: neurological outcome at two years in a cohort of relapsing-remitting patients. Funct Neurol. 2012;27 (1):55-59.

28. Paul F, Wattjes MP. Chronic cerebrospinal venous insufficiency in multiple sclerosis: the final curtain. Lancet. 2014;383(9912):106-108.

29. Chalmers I. Unbiased, relevant, and reliable assessments in health care: important progress during the past century, but plenty of scope for doing better. BMJ. 1998;317(7167):1167-1168. 